\title{
Expanding landscapes of the diversified mcr-1-bearing plasmid reservoirs
}

\author{
Qingjing Wang ${ }^{1 \dagger}$, Jian Sun ${ }^{2 \dagger}$, Jun $\mathrm{Li}^{3+}$, Youfa Ding ${ }^{4}$, Xing-Ping Li ${ }^{2}$, Jingxia Lin ${ }^{1}$, Bachar Hassan ${ }^{5}$ and Youjun Feng ${ }^{1 *}$
}

\begin{abstract}
Background: Polymyxin is a cationic polypeptide antibiotic that can disrupt bacterial cell membrane by interacting with its lipopolysaccharide molecules and is used as a last resort drug against lethal infections by the carbapenemresistant superbugs (like NDM-1). However, global discovery of the MCR-1 colistin resistance dramatically challenges the newly renewed interest in colistin for clinical use.

Methods: The mcr-1-harboring plasmids were acquired from swine and human Escherichia coli isolated in China, from 2015 to 2016, and subjected to Illumina PacBio RSII and Hi-Seq2000 for full genome sequencing. PCR was applied to close the gap of the assembled contigs. Ori-Finder was employed to predict the replication origin (oriC) in plasmids. The phenotype of MCR-1-producing isolates was evaluated on the LBA plates with various level of colistin. Genetic deletion was used to test the requirement of the initial "ATG" codon for the MCR-1 function.

Results: Here, we report full genomes of over $10 \mathrm{mcr}$-1-harboring plasmids with diversified replication incompatibilities. A novel hybrid IncI2/IncFIB plasmid pGD17-2 was discovered and characterized from a swine isolate with colistin resistance. Intriguingly, co-occurrence of two unique mcr-7-bearing plasmids (pGD65-3, Incl2, and pGD65-5, IncX4) was detected in a single isolate GD65, which might accelerate dissemination of the mcr-1 under environmental selection pressure. Genetic analyses of these plasmids mapped mobile elements in the context of antibiotic resistance and determined two insertion sequences (ISECPI and ISAp/1) that are responsible for the mobilization of mcr-1. Gene deletion also proved that the first ATG codon is redundant in the mcr-1 gene.
\end{abstract}

Conclusions: Collectively, our results extend landscapes of the diversified mcr-1-bearing plasmid reservoirs.

Keywords: Landscape, Plasmid genome, mcr-1, Colistin resistance, Dissemination

\section{Background}

Antibiotics play crucial roles in controlling the dissemination of lethal infections by bacterial pathogens. However, the emergence of antibiotic resistance is developing a great threat to public health worldwide. It is estimated that multidrug-resistant (MDR) pathogens cause nearly 700,000 cases of lethal infections (including 214,000 neonatal deaths) each year [1]. The discovery of New Delhi $\beta$-lactamase 1 (NDM-1) and its variants like NDM-5 had ever pushed us on the cusp of postantibiotic era, in that they can confer potent resistance to extended spectrum $\beta$-lactamases (ESBL) and carbapenems, the two classes of widely used antibiotics

\footnotetext{
* Correspondence: fengyj@zju.edu.cn

${ }^{\dagger}$ Equal contributors

${ }^{1}$ Department of Medical Microbiology and Parasitology, Zhejiang University

School of Medicine, Hangzhou 310058, Zhejiang, China

Full list of author information is available at the end of the article
}

against the MDR bacteria [2, 3]. In general, the cationic polypeptide antibiotic polymyxin E (colistin) is believed to act as a last resort against fatal infections by gramnegative pathogens with pan-drug resistance $[4,5]$. However, it seems likely that gut bacteria have evolved some mechanisms to bypass the final line of refuge antibiotic (i.e., colistin resistance) [4-7]. Biochemical mechanism by which bacteria acquire the appreciable resistance to colistin is mainly dependent on the reduction in the net negative charge of the bacterial outermembrane, which consequently decreases the binding affinity of colistin to bacterial surface [8-10]. To the best of our knowledge, four types of modifications of the lipid A moiety anchored on bacterial lipid polysaccharide (LPS) are involved in the alteration of the net negative surface charge, which include (i) modification at 3 'linked second fatty acyl chain with glycine (and/or 
diglycine) [8]; (ii) the addition of amino-arabinose to 4'phosphate position of sugar [9]; (iii) the addition of sugar with phosphoethanolamine (pEtN) at 4'-phosphate position [10, 11]; and (iv) the attachment of galactosamine (GalN) to 1'-phosphate of sugar [12, 13]. Unlike the fact that a single ArnT enzyme catalyzes the reaction for amino-arabinose-based sugar modification of lipid A in Pseudomonas aeruginosa ( $P$. aeruginosa) [14] and Cupriavidus metallidurans [9], a threecomponent system (AlmG-AlmF-AlmE, encoded by an operon of Vc1577-Vc1578-Vc1579) is evolved to modify the lipid A with glycine in Vibrio cholerae [8]. The pEtN modification of lipid A originally found in Klebsiella pneumoniae ( $K$. pneumoniae) is attributed to the point mutations of the phoP-phoQ two-component system and its regulator gene $\operatorname{mgr} B$ [11]. Intriguingly, the modifications of lipid A with both GalN and pEtN account for colistin resistance in the serious opportunistic pathogens Acinetobacter baumannii (A. baumannii) [13, 15]. Additionally, the PmrA/PmrB two-component system is also implicated into genetic control of lipid A modification as well as polymyxin resistance in Salmonella enterica ( $S$. enterica) [16], A. baumannii, and P. aeruginosa [14]. Thus, we anticipated that enteric pathogens have developed multiple mechanisms to remodel its lipid A structure/integrity on the LPS under dynamic selection by the altered environments, like antibiotic exposures.

The emergence of the mobilized colistin resistance gene $(m c r-1)$ illustrated a new mechanism for plasmidborne polymyxin resistance [6]. The protein product of $m c r-1$, referred to MCR-1, is annotated as an integral membrane protein with five trans-membrane helices [7]. Our genetic experiment proved that localization of the MCR-1 in bacterial peri-plasm is critical for its role in colistin resistance through deleting the trans-membrane region [7]. Together with catalytic structures of MCR-1 reported by three other research groups [17-19], our structure-guided functional studies revealed the requirement of a five-residue-containing motif for its biochemical function [7]. Since its first discovery in Southern China in the late of 2015 [6], the $m c r-1$ gene have been detected in nearly 40 countries from five of seven continents $[7,20]$. The $m c r-1$-positive gut bacteria included Escherichia coli (E. coli) [21, 22], S. enterica [23, 24], $K$. pneumoniae [6, 25, 26], Enterobacter aerogenes (E. aerogenes) [27], Kluyvera ascorbata [28], Citrobacter freundii [29], and Citrobacter braakii [30]. Not only does the $m c r-1$ gene appear in diversified $E$. coli isolates with different sequence types $[20,31]$, but also it coexists with the NDM-1 $[32,33]$ and its variants, like NDM-5 [34, 35] and NDM-9 [36]. Additionally, ESBL can be coproduced with MCR-1 from a single plasmid or a same bacterial isolate [37-39]. Further sequence analyses showed that the mcr-1-harboring plasmid reservoirs exhibit an unexpected diversity [20-22]. Multiple lines of replication incompatibility have been assigned to these $m c r$-1-bearing plasmids, which included IncP [26], IncI2 [6, 23, 24, 35, 40, 41], IncX1-X2 mosaic version [42], IncX3-X4 hybrid type [43], IncX4 [7, 44, 45], IncHI2 [23, 24, 35, 46], IncFI [47], IncFII [48], and IncHI1 [49], respectively. Obviously, it seems likely that the spread of $m c r-1$ proceeds by complex dissemination across diversified species among Enterobacteriaceae.

Our recent study suggested that a group of $\mathrm{mcr}-1$ positive plasmids with distinct backbones are present in swine farm $E$. coli isolates as well as human clinical $E$. coli isolates [20]. Here, we report comparative genomics of over 10 representative $m c r-1$-bearing plasmids. Unexpectedly, we identified a new mcr-1-carrying hybrid plasmid pGD17-2 classified into two different replication incompatibilities (IncFIB and IncI2). Moreover, we are first to observe coexistence of two unique $\mathrm{mcr}$-1-positive plasmids (IncI2-type pGD65-3 and IncX4-like pGD65-4) in a same $E$. coli isolate. In addition, we determined a couple of variants of the other known mor-1-positive plasmids. These findings expand greatly the landscape of mor-1-harboring plasmids.

\section{Methods \\ Conjugation, S1-PFGE, and Southern hybridization}

The $m c r$-1-positive samples we obtained were kept in LB broth or on LB agar with appropriate antibiotics. $E$. coli C600 was used as the recipient for the conjugation experiment of MCR-producing $E$. coli isolates. The trans-conjugants were selected on MacConkey agar containing colistin $(2 \mu \mathrm{g} / \mathrm{ml})$ and streptomycin $(2000 \mu \mathrm{g} / \mathrm{ml})$ and finally confirmed by PCR and ERIC-PCR. To analyze the location of the $m c r-1$ gene, S1-PFGE and Southern blot analysis were performed using the original strains and trans-conjugants carrying $\mathrm{mcr}^{-1}$ gene. Briefly, whole-cell DNA of the E. coli strains were extracted and embedded in agarose gel plugs. Subsequently, the agarose gel plugs were treated with $S 1$ nuclease (TaKaRa, Dalian, China), and the DNA fragments were separated by PFGE. Southern blot hybridization was then performed with the digoxigeninlabeled probes (Roche Diagnostics, Mannheim, Germany) specific for the $m c r-1$ gene.

\section{Bacterial samples, plasmid sequencing, and sequence assembly}

The plasmids and primers used in this study are listed in Additional file 1: Tables S1 and S2. Fourteen plasmids were sequenced by Illumina PacBio RSII and $\mathrm{Hi}$ Seq2000 using prepared paired-end $2 \times 200$-bp libraries, of which raw data were separately assembled using SOAP de novo (version 2.0.4) and SMRT Analysis (version 2.2.0), after high-quality read acquisition, and any 
assembly discrepancies/uncertainties filled or closed with PCR. The finally generated contigs were checked for circularization, whereas overlapping ends were trimmed. The general features of the 14 plasmids are summarized in Additional file 1: Table S1. Ori-Finder was employed to predict the replication origin (oriC) in plasmids under study [50]. The assembled plasmid sequences were annotated by using the RAST suite for the identification of protein-coding sequences, and ARAGORN [51] for tRNA, and RNAmmer [52] for rRNA.

\section{Annotation of plasmids and resistance genes}

A bacterial mobilome annotation procedure [53] was designed to type sequenced/assembled plasmids and to identify insertion sequences (ISs), integrons, and prophages. The $m c r-1$ gene has been proposed to confer colistin resistance, while other mobile-related elements were detected using specific informatics tools. PlasmidFinder [54] was used to type replicons of plasmids that had been previously assembled and identified. ISs were mainly identified with ISsaga [55] and ISfinder [56] and then manually adjusted with focuses on intactness, terminal inverse repeats (IRs), and flanking direct repeats (DRs). ISCR elements were also identified (http://www.cardiff.ac.uk/research/explore/research-units/ antibacterial-agents-and-genetics-of-resistance). Prophages were predicted with PHAST [57] and Phage_Finder [58]. Resistance genes were in silico identified with ARDB [59], CARD [60], and ResFinder [61].

\section{Determination of colistin resistance}

According to the recommendation of EUCAST, microbroth dilution method was used to address colistin MIC, whose level of $\leq 0.25 \mu \mathrm{g} / \mathrm{ml}$ was treated susceptible. For functional evaluation of $m c r-1$ derivatives in colistin resistance, agar dilution method was applied and resistance level of the colistin-susceptible strain MG1655 $(\leq 2.0 \mu \mathrm{g} / \mathrm{ml})$ is the cutoff value.

\section{Nucleotide sequence accession number}

The plasmid nucleotide sequences reported in this study have been deposited in GenBank under accession number listed in Additional file 1: Table S1.

\section{Results and discussion}

Genetic evidence for the presence of $\mathrm{mcr}-1$ in diversified plasmids

Our recent study reported hundreds of $m c r$-1-positive plasmids through extensive dissection of a thousand of $E$. coli isolates from swine farm and hospitalized patients, which implied that the diversified $m c r-1$-carrying plasmids are present [20]. In this follow-up study, 13 representative mcr-1-positive plasmids (Additional file 1: Table S1) were subjected to further genetic analyses of both S1-pulsed- field gel electrophoresis (PFGE) (Additional file 2: Figure $\mathrm{S} 1 \mathrm{~A}$ and $\mathrm{C}$ ) and Southern hybridization blot (Additional file 2: Figure S1B and D). As expected, diversified plasmids were present in the above $13 \mathrm{E}$. coli isolates (Additional file 2: Figure S1). Of note, ten trans-conjugants were successfully obtained from the $13 \mathrm{mcr}$-1-positive isolates. Southern blotting revealed that the $m c r-1$ gene was present on plasmids ranging from $\sim 33$ to $240 \mathrm{~kb}$ (Additional file 2: Figure S1). First, it was estimated that no less than three isolates (GD46, GD65, and WH03) carries the $\mathrm{mcr}$-1-positive plasmid of $\sim 33 \mathrm{~kb}$ long (Additional file 2: Figure $\mathrm{S} 1 \mathrm{~B}$ and $\mathrm{D}$ ). Second, the $m c r-1$ gene is located on $\sim 60 \mathrm{~kb}$ plasmid in seven isolates (Additional file 2: Figure S1B and D). Third, two big $m c r$-1-harboring plasmids ( 120 kb for pGD17-2 and $\sim 240 \mathrm{~kb}$ for pGD802) separately occurred in the strains GD17-2 and GD80-2 (Additional file 2: Figure S1B and D). Although we failed to detect $m c r$-1-positive band in the strain Lishui12 using Southern blot (Additional file 2: Figure S1B), we proved that it is PCR-positive for $m c r-1$, and had a success in isolating a mcr-1-producing plasmid pLishui12 of $\sim 33 \mathrm{~kb}$ long (Additional file 2: Figure S4). Curiously, in the strain GD65, the $m c r-1$ gene was found on two distinct plasmids with different sizes ( $\sim 30 \mathrm{~kb}$ for pGD65-4 and $\sim 60 \mathrm{~kb}$ for pGD65-3, Additional file 2: Figure S1B). The physical evidence for the presence of $m c r-1$ in the above plasmids was validated by further PCR analyses combined with direct DNA sequencing. All the above $m c r-1$-positive plasmids can confer the E. coli strains to possess modest level of colistin MIC [2-4 $\mu \mathrm{g} / \mathrm{ml}]$ (Table 1).

\section{Overall landscape of the mcr-1-carrying plasmids}

In total, full replicon sequences of $13 \mathrm{mcr}$-1-harboring plasmids were assembled through $\mathrm{Hi}$-Seq sequencing (Illumina Inc.), in which the gaps were closed with ABI 3730 Sanger sequencing (Additional file 1: Table S1). Using specific primers (Additional file 1: Table S2), all these plasmids were confirmed by the close-loop PCR (Additional file 2: Figure S8A). BLAST-based query using PlasmidFinder (https://cge.cbs.dtu.dk/services/ PlasmidFinder/) revealed that the newly-determined 14 plasmids are classified into four types as follows: eight IncI2-type plasmids, four IncX4-like plasmids, one IncHI2-type, and one IncI2/IncFIB hybrid plasmid pGD17-2 (Additional file 1: Table S1 and Additional file 2: Figures S1-S7). It is noted that (i) the insertion sequence ISApl1 is located immediately upstream of the $m c r-1$ gene (Figs. 1 and 2) and (ii) a putative locus pap 2 encoding the PAP2-family protein is neighbored with the downstream of the $m c r-1$ gene (Fig. 2), which might facilitate the transfer of $m c r-1$ [39]. We also noted that the insertion sequence of ISApl1 is lacking in some cases, implying a relic for frequent conjugation of ISApl1-mcr-1-pap2. Therefore, the discovery of these plasmids significantly contributed 
Table 1 Colistin MIC of clinical E. coli strains expressing $\mathrm{mcr}-1$

\begin{tabular}{|c|c|c|c|c|c|c|c|c|c|c|c|c|c|}
\hline & Strains & & & & & & & & & & & & \\
\hline \multirow[t]{2}{*}{ Colistin MIC $(\mu \mathrm{g} / \mathrm{ml})$} & GD17 & GD65 & GD80 & GD23 & GD53 & LS142 & WH07 & WH09 & WH13 & GD81 & LS12 & GD46 & WHO3 \\
\hline & 2 & 4 & 4 & 2 & 2 & 4 & 4 & 4 & 4 & 2 & 4 & 4 & 4 \\
\hline
\end{tabular}

to a growing collection of $m c r-1$-positive plasmids with known replicon sequences (Additional file 1: Table S3).

Briefly, in similarity to the newly identified plasmid pLishui142-1 (61.908 kb), seven additional new members of IncI2-type $\mathrm{mcr}$-1-bearing plasmids were determined in this study, which corresponded to pGD23-3 (64.078 kb), pGD53-3 (62.813 kb), pGD65-3 (64.857 kb), pGD81-1 (62.712 kb), pWH07-3 (62.067 kb), pWH09-3 (62.072 kb), and pWH13-4 (60.694 kb), respectively (Additional file 1: Table S1). Although this type of plasmids lacks the insertion sequence ISApl1 (Additional file 2: Figure S2), they consistently share a site-specific recombinase $(r c i)$ gene, two pilus-encoding genes (pil and tra), and four different pilli proteins (Additional file 2: Figure S2). In addition, we found that heterogeneity of whole-shufflon structural variations exists between the 3 ' region of pilV gene and $r c i$ gene in $m c r-1$-harboring IncI2 plasmids as recently reported [62]. The plasmid sequences yielded the following results: in pWH9-3 and pWH7-3, rci-A-A $-\mathrm{C}-\mathrm{C}$ '-B'-D'-pilV; in pWH13-4, rci-A-A'-C-C'-D'-B'-pilV; in LS142-1, rci-B'-D'-A-A'-pilV; in 65-3, rci-A'-A-D'-B'-C$C^{\prime}-B^{\prime}-D^{\prime}-A-A^{\prime}-p i l V$; in pGD23-3, rci-C-C'-D'-B'-A'-A-B '-D'-pilV; in pGD53-3, rci-C-C'- $\mathrm{B}^{\prime}-\mathrm{D}^{\prime}-\mathrm{D}^{\prime}-\mathrm{B}^{\prime}-\mathrm{A}^{\prime}-\mathrm{A}-$ pill; and in hybrid plasmid pGD17-2, rci-C-C'-A-A'-D'-B '-pilV. Unlike the scenario seen with the IncHI2 plasmid pHNSHP45-2 that carries the $m c r-1$ gene [46], the typical $m c r-1$ cassette is located in different locations of pGD80-2
(241.033 kb), a member of the IncHI2-type plasmid family (Additional file 2: Figure S3). Moreover, it encodes three more resistance genes $\left(\operatorname{aph}\left(3^{\prime}\right)-I a, o q x A B\right.$, and tetR), suggesting complexity/flexibility among the $m c r-1$-bearing IncHI2 plasmids.

In addition to pLishui12 (33.309 kb) [63], three more $m c r$-1-harboring IncX4 plasmids we reported here included pGD46-3 (33.302 kb), pGD65-4 $(33.305 \mathrm{~kb})$, and pWH03 (33.292 kb), respectively (Additional file 1: Table S1). All the four IncX4 plasmids are featuring the almost identical genetic backbone in which no ISApl1 element is flanked by the only antibiotic resistance gene $\mathrm{mcr}-1$ gene (Additional file 2: Figures S4, S5, and S7). Genome alignments indicated that plasmid pGD46-3 was nearly identical to the plasmid pMCR1_IncX4 (Accession no.: KU761327 from $K$. pneumoniae in China). It seems likely that pGD46-3 might be generated through the integration of the $m c r-1$ gene into the specific site between the pir and parA genes of IncX4-type pSH146_32 plasmid (Additional file 2: Figure S5).

\section{Discovery of a new mcr-1-positive hybrid plasmid pGD17-2}

The novel $m c r-1$-positive plasmid pGD17-2 we determined (Fig. 1 and Additional file 1: Table S1) allows the strain GD17 to grow well on the LBA plate

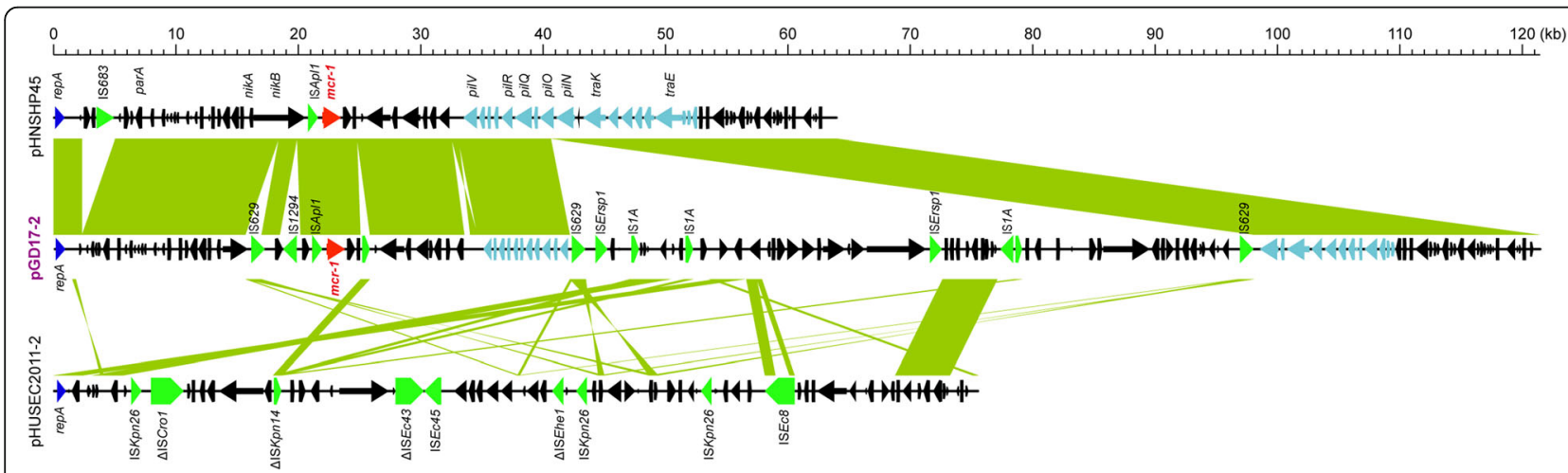

Fig. 1 Discovery of a new hybrid plasmid pGD17-2 that comprises the mcr-1 gene. The full genome of pGD17-2 is sequenced and collinearly compared with the counterpart of pHNSHP45 and pHUSEC2011-2. The mosaic plasmid pGD17-2 (highlighted in purple) is isolated from a colistinresistant strain GD17. The mcr-1-positive Incl2-type plasmid pHNSHP45 and the mcr-1-negative plasmid pHUSEC2011-2 both are applied to define the plasmid backbone of pGD17-2, giving the presence of two incompatible replicon types (IncFIB and Incl2). The relevant parts of plasmid are also shown to highlight the syntenic regions. Regions of synteny between adjacent schematics are indicated by the shaded areas. These schematics are drawn to scale. Genes associated with the tra and pil loci are indicated by light blue arrows, while replication-associated genes are denoted as dark blue arrows. The mcr-1 gene is indicated with the red arrow, while accessory genes are indicated by black arrows. Insertion sequences are highlighted in green arrows 


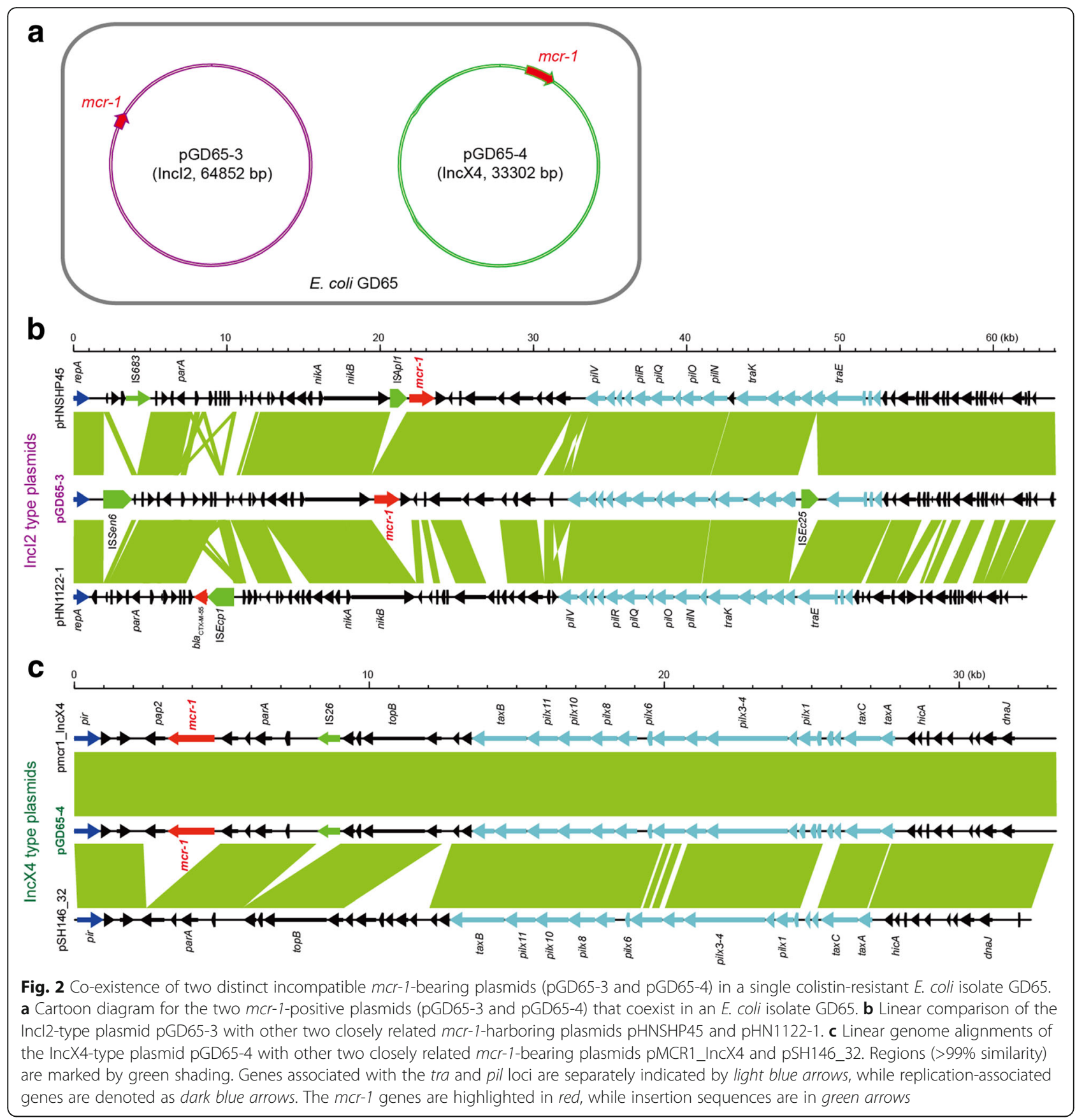

supplemented with up to $16 \mathrm{mg} / \mathrm{L}$ of colistin (Additional file 2: Figure S9). The plasmid size of pGD17-2 is $121.454 \mathrm{~kb}$ (Additional file 1: Table S1), whose GC\% is $43.69 \%$. To rule out the possible errors in plasmid sequencing or assembly, hybrid plasmid pGD17-2 was further confirmed by subsequent PCR analysis and Sanger sequencing (Additional file 2: Figure S8 A and B). Comparative genomics revealed that pGD17-2 is a new hybrid one featuring with two types of plasmid backbones (IncI2 and IncFIB, in Fig. 1), implying temporary transition status of $m c r-1$ transmission or genetic stability under special selection pressure. Relative to the paradigm $m c r$-1-harboring IncI2-type plasmid pHNSHP45, pGD17-2 exhibited 51\% coverage and 99\% identity and possessed two insertion sequences (IS629 and IS1294) in the $n i k B$ gene, as well as an additional IS629-mediated insertion sequence $(\sim 56 \mathrm{~kb})$ in the pilO gene (Fig. 1 and Additional file 2: Figure S2). Further sequence alignments suggested that the above insertion sequence $(\sim 56 \mathrm{~kb})$ has only $26 \%$ coverage to a $m c r-1$-negative plasmid pHUSEC2011-2 (Accession no.: HE610901) (Fig. 1 and Additional file 2: Figure S2). Notably, the 
mobile genetic elements identified from the pGD17-2 plasmid are highly related to the cognate conjugationassociated counterparts or remnants (a syntenic F-like or R-like type IV secretion system gene clusters and a relaxase gene traI (Fig. 1 and Additional file 2: Figure S2). In comparison with all the mcr-1-carrying plasmids (Additional file 1: Table S3), pGD17-2 represents a new hybrid plasmid producing MCR-1 colistin resistance.

\section{Co-occurrence of two unique mcr-1-harboring plasmids (pGD65-3 and pGD65-4) in a single $E$. coli isolate}

Unexpectedly, two $m c r-1$-bearing plasmids namely pGD65-3 and pGD65-4 (Fig. 2a and Additional file 2: Figure S1) were localized onto a single $E$. coli strain GD65 with significant colistin resistance (Additional file 2: Figure S9). The co-occurrence of these two plasmids was experimentally validated with Southern blot (Additional file 2: Figure S1B). Sequencing results showed that pGD65-3 (33.301 kb in length) is an IncI2type plasmid, whereas the other one pGD65-4 (64.852 $\mathrm{kb}$ in length) is featuring a IncX4-type plasmid backbone. In similarity to all the other known IncI2-type plasmids, no insertion sequence ISApl1 is neighbored with the $m c r-1$ gene in the pGD65-3 plasmid (Fig. 2b and Additional file 2: Figure S6). Although the IncX4like plasmid pGD65-4 lacks the ISApl1 insertion sequence in front of the $m c r-1$, but it retains pap 2 at the 3 '-end of the $m c r-1$ locus (Fig. 2c, Additional file 2: Figures S4, S5 and S7). Conjugation-based PCR detection experiments demonstrated that the two plasmids (pGD65-3 and pGD65-4, in Fig. 2) can be separately transferred from the donor strain E. coli GD65 (Additional file 2: Figure S8C) to the recipient E. coli strain C600 (Additional file 2: Figure S8D) and give appreciable level of resistance to colistin $(\sim 16 \mu \mathrm{g} / \mathrm{ml})$. Together, this result defined a paradigm for coexistence of two unique $m c r-1$-harboring plasmids (pGD65-4, IncX4 type, and pGD65-3, IncI2 type) in a single $E$. coli isolate.

\section{Conclusions}

The data presented here extends landscapes of $\mathrm{mcr}-1$ positive plasmids and highlights complexity of MCR-1 colistin resistance in gut microbiota (Fig. 3). Unlike the scenarios seen with the first reported $\mathrm{mcr}$-1-harboring plasmid pHNSHP45, there are no ISApl1 element in front of the $m c r-1$ gene in all the eight Incl2 plasmids we reported (pGD23-3, pGD53-3, pGD65-3, pGD81-1, pLishui142-1, pWH07-3, pWH09-3, and pWH13-4, in Additional file 2: Figure S7A). It is noted that all the IncI2 plasmids consistently contain a plasmid-borne site-specific recombinase gene, $r c i$ (Additional file 2: Figure S7A). Variations exist in the 3 '-end sequence of pap2 gene on IncI2 plasmids, of which the three plasmids (pWH07-3, pWH09-3, and pWH13-4) are almost

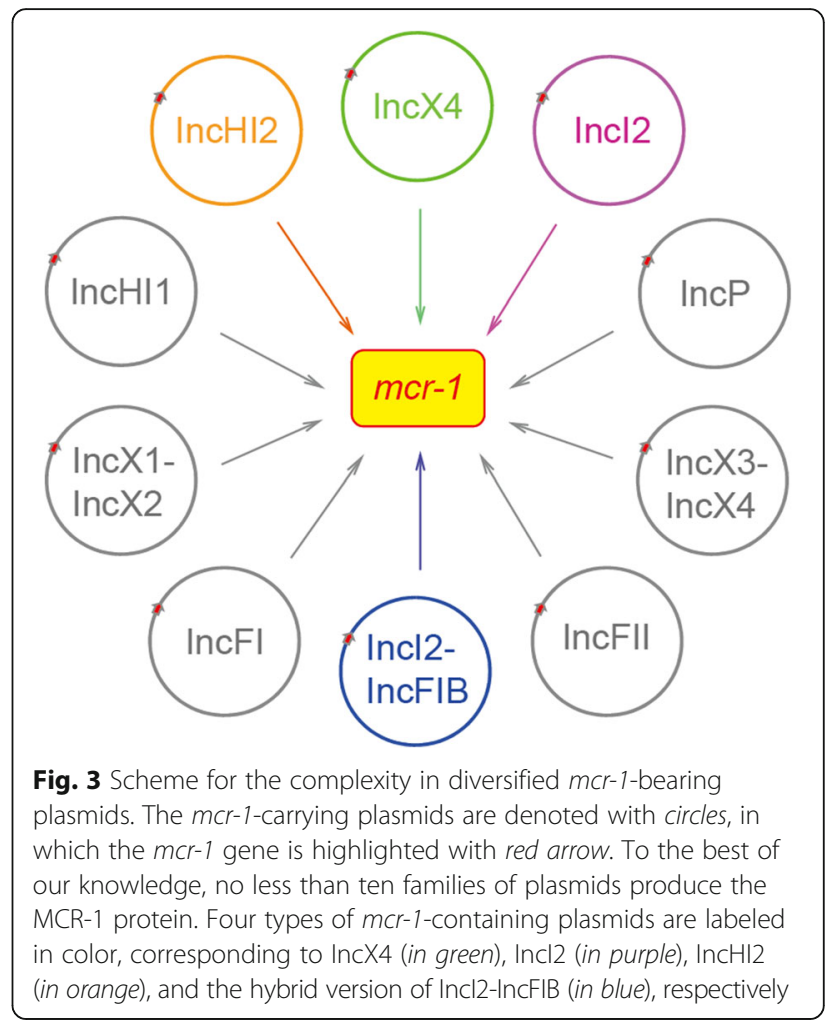

the same and the other three plasmids (pGD23-3, pGD53-3 and pGD65-3) are highly identical (Additional file 2: Figure S7A). By contrast, the mosaic plasmid pGD17-2 is more flexible, with IS629 and IS1294 insertion in the nikB gene. And ISApl1 immediately appears in front of $m c r-1$ in pGD17-2. Thus, the hybrid plasmid pGD17-2 represents a novel mobile element carrying mcr-1 and provides remarkable insights into the plasmid-mediated transfer of MCR-1 colistin resistance.

In the mcr-1-harboring IncX4 plasmids (pGD46-3, pGD65-4, Lishui12, and pWH03), the regions between parA gene and repA gene are nearly identical (Additional file 2: Figure S7B), in that they shared 99\% similarity to IncX4 mcr-1-harboring K. pneumoniae plasmid pMCR1_IncX4 (Accession no.: KU761327). In comparison with the IncX4 plasmid pSH146_32, the mcr-1 gene was found to be inserted in the same location. Different with $m c r$-1-harboring IncI2 plasmids, the 3 '-end sequences of pap 2 are consistently present in IncX4 plasmids. In addition, no ISApl1 elements were found to flank the $m c r-1$ gene (Additional file 2: Figure S7B). The fact that strain GD65 carries two $m c r-1$-positive plasmids (pGD65-3 and pGD65-4) might represent a first example that both IncI2-type plasmid pGD65-3 and IncX4-type plasmid pGD65-4 contributed to colistin resistance in $E$. coli. Retrospectively, the two $\mathrm{mcr}$-1-harboring plasmids (IncI2-like plasmid pHNSHP45 [6] and IncHI2-type plasmid pHNSHP45-2 [46]) from the E. coli strain SHP45 
constitute an additional example. However, it seems likely the coexistence of two different $m c r$-1-positive plasmids does not confer distinguishable ability of the recipient strains in colistin resistance when compared with the strain with a single $m c r-1$-plasmid (Additional file 2: Figure S9). In general, two successive ATG codons initiate the $m c r-1$ gene in most of the cases like GD17 and GD65. However, we noted that only one ATG in the case of strain Lishui142 (abbreviated as LS142). We therefore tested the function of this $m c r-1$ variant [referred to $\mathrm{mcr}$ $1(\Delta 1)$ ] using the pBAD24 expression system (Additional file 2: Figure S9). In fact, it gave indistinguishable ability when compared to the normal version $m c r-1$ in the trials of colistin resistance, suggesting the redundancy of one "ATG" codon in the MCR-1.

In summary, no less than ten types of plasmids mediate the transmission of MCR-1 colistin resistance in field and clinical isolates (Fig. 3). Our results extended significantly our understanding of diversified $\mathrm{mcr}$-1-bearing plasmids, whose dissemination constitutes a potential threat to public health and clinical treatment. Global distribution of $m c r-1$ in E. coli populations emphasizes the importance to prevent global over- and/or mis-use of colistin. Extensive surveillance programs of antibiotic resistance should be extended to swine farms, and even in human medicine, since $m c r-1$ has been recently identified in human isolates. Restrictive/rational use of colistin is urgently required to prevent the rapid spread of $\mathrm{mcr}-1$ to other bacteria and in different niches, excluding human hospitals and foodborne settings.

\section{Additional files}

Additional file 1: Table S1. New mcr-1-harboring plasmids with full genomes sequenced in this study. Table S2. Primers used in this study. Table S3. List of mcr-1-positive plasmids with known genomes. (DOC $237 \mathrm{~kb}$ )

Additional file 2: Figure S1. Genetic evidence for the diversified $\mathrm{mcr}^{-1}$ positive plasmids. Figure S2. Comparative genomics of the eight Incl2 type mcr-1-carrying plasmids. Figure $\mathbf{S 3}$. Genome comparison of the pGD65-3 plasmid with the mcr-1-carrying plasmid pHNSHP45-2. Figure S4. Co-linear genome alignments for the four IncX4 type mcr-1-carrying plasmids. Figure S5. Genomic comparison of the newly determined plasmid pGD46-3 with the recently reported one pmcr-1_IncX4 and the mcr-1-lacking plasmid pSH146_32. Figure S6. Genomic analyses for the three Incl2-type plasmids. Figure S7. Fine mapping of the mcr-1-surrounding regions. Figure S8. Genetic identification and characterization of the fourteen $\mathrm{mCr}$-1-harboring plasmids. Figure S9. Measurement of the ability of clinical (and/or engineered) E. coli strains in colistin resistance. (DOC $3018 \mathrm{~kb}$ )

\section{Abbreviations}

A. baumannii: Acinetobacter baumannii; ESBL: Extended spectrum $\beta$ lactamases; GalN: Galactosamine; K. pneumoniae: Klebsiella pneumoniae; $\mathrm{mcr}$ 1: Mobilized colistin resistance gene; MDR: Multidrug-resistant; NDM-1: New Delhi $\beta$-lactamase 1; P. aeruginosa: Pseudomonas aeruginosa; pEtN: Phosphoethanolamine; S. enterica: Salmonella enterica

\section{Acknowledgements}

Not applicable

\section{Funding}

This work was supported by the National Key Basic Research Program of China (2016YFC1200100 \& 2017YFD0500202). Dr. Feng is a recipient of the "Young 1000 Talents" Award.

\section{Availability of data and materials}

Not applicable

\section{Authors' contributions}

YF designed the project; YF, JS, QW, JL, YD, XL, and J-X L performed experiments; $\mathrm{YF}, J \mathrm{~S}, \mathrm{JL}$, and $\mathrm{QW}$ analyzed the data and prepared the figures; YF, JS, JL, and HB drafted this manuscript. All authors read and approved the final manuscript.

Ethics approval and consent to participate

Not applicable

Consent for publication

Not applicable

Competing interests

The authors declare that they have no competing interests.

\section{Publisher's Note}

Springer Nature remains neutral with regard to jurisdictional claims in published maps and institutional affiliations.

\section{Author details}

${ }^{1}$ Department of Medical Microbiology and Parasitology, Zhejiang University School of Medicine, Hangzhou 310058, Zhejiang, China. ${ }^{2}$ National Risk Assessment Laboratory for Antimicrobial Resistance of Animal Original Bacteria, College of Veterinary Medicine, South China Agricultural University, Guangzhou 510642, Guangdong, China. ${ }^{3}$ Key Laboratory of Bioorganic Synthesis of Zhejiang Province, College of Biotechnology and Bioengineering, Zhejiang University of Technology, Hangzhou 310014, Zhejiang, China. ${ }^{4}$ Lishui People's Hospital, Lishui 323000, Zhejiang, China. ${ }^{5}$ University of North Carolina, Chapel Hill, NC, USA.

Received: 23 January 2017 Accepted: 19 June 2017

Published online: 06 July 2017

\section{References}

1. Laxminarayan R, Amabile-Cuevas CF, Cars O, Evans T, Heymann DL, et al. UN High-Level Meeting on antimicrobials - what do we need? Lancet. 2016; 388:218-20.

2. Kumarasamy KK, Toleman MA, Walsh TR, Bagaria J, Butt F, et al. Emergence of a new antibiotic resistance mechanism in India, Pakistan, and the UK: a molecular, biological, and epidemiological study. Lancet Infect Dis. 2010;10:597-602.

3. Hornsey M, Phee L, Wareham DW. A novel variant, NDM-5, of the New Delhi metallo-beta-lactamase in a multidrug-resistant Escherichia coli ST648 isolate recovered from a patient in the United Kingdom. Antimicrob Agents Chemother. 2011;55:5952-4.

4. Schwarz S, Johnson AP. Transferable resistance to colistin: a new but old threat. J Antimicrob Chemother. 2016;71:2066-70.

5. Baron S, Hadjadj L, Rolain JM, Olaitan AO. Molecular mechanisms of polymyxin resistance: knowns and unknowns. Int J Antimicrob Agents. 2016. doi:10.1016/j.jantimicag.2016.06.023. [Epub ahead of print].

6. Liu YY, Wang Y, Walsh TR, Yi LX, Zhang R, et al. Emergence of plasmidmediated colistin resistance mechanism MCR-1 in animals and human beings in China: a microbiological and molecular biological study. Lancet Infect Dis. 2016;16:161-8.

7. Gao R, Hu Y, Li Z, Sun J, Wang Q, et al. Dissemination and mechanism for the MCR-1 colistin resistance. PLoS Pathog. 2016;12:e1005957. doi:10.1371/ journal.ppat.1005957. eCollection 2016 Nov.

8. Hankins JV, Madsen JA, Giles DK, Brodbelt JS, Trent MS. Amino acid addition to Vibrio cholerae LPS establishes a link between surface remodeling in gram-positive and gram-negative bacteria. Proc Natl Acad Sci U S A. 2012; 109:8722-7. 
9. Petrou VI, Herrera CM, Schultz KM, Clarke OB, Vendome J, et al. Structures of aminoarabinose transferase ArnT suggest a molecular basis for lipid A glycosylation. Science. 2016;351:608-12.

10. Sun J, Xu Y, Gao R, Lin J, Wei W, et al. Deciphering MCR-2 colistin resistance. MBio. 2017;8. doi: 10.1128/mBio.00625-17.

11. Cannatelli A, D'Andrea MM, Giani $T$, Di Pilato $V$, Arena $F$, et al. In vivo emergence of colistin resistance in Klebsiella pneumoniae producing KPC-type carbapenemases mediated by insertional inactivation of the PhoQ/PhoP mgrB regulator. Antimicrob Agents Chemother. 2013;57: 5521-6.

12. Llewellyn AC, Zhao J, Song F, Parvathareddy J, Xu Q, et al. NaxD is a deacetylase required for lipid A modification and Francisella pathogenesis. Mol Microbiol. 2012;86:611-27.

13. Chin CY, Gregg KA, Napier BA, Ernst RK, Weiss DS. A PmrB-regulated deacetylase required for lipid A modification and polymyxin resistance in Acinetobacter baumannii. Antimicrob Agents Chemother. 2015;59:7911-4.

14. McPhee JB, Lewenza S, Hancock RE. Cationic antimicrobial peptides activate a two-component regulatory system, $\mathrm{PmrA}$-PmrB, that regulates resistance to polymyxin B and cationic antimicrobial peptides in Pseudomonas aeruginosa. Mol Microbiol. 2003;50:205-17.

15. Beceiro A, Llobet E, Aranda J, Bengoechea JA, Doumith M, et al. Phosphoethanolamine modification of lipid A in colistin-resistant variants of Acinetobacter baumannii mediated by the pmrAB two-component regulatory system. Antimicrob Agents Chemother. 2011;55:3370-9.

16. Tamayo R, Ryan SS, McCoy AJ, Gunn JS. Identification and genetic characterization of PmrA-regulated genes and genes involved in polymyxin B resistance in Salmonella enterica serovar typhimurium. Infect Immun. 2002; 70:6770-8.

17. Stojanoski V, Sankaran B, Prasad BV, Poirel L, Nordmann P, et al. Structure of the catalytic domain of the colistin resistance enzyme MCR-1. BMC Biol. 2016;14:81.

18. Ma G, Zhu Y, Yu Z, Ahmad A, Zhang H. High resolution crystal structure of the catalytic domain of MCR-1. Sci Rep. 2016;6:39540.

19. Hu M, Guo J, Cheng Q, Yang Z, Chan EW, et al. Crystal structure of Escherichia coli originated MCR-1, a phosphoethanolamine transferase for colistin resistance. Sci Rep. 2016;6:38793.

20. Wang Q, Li Z, Lin J, Wang X, Deng X, et al. Complex dissemination of the diversified mcr-1-harbouring plasmids in Escherichia coli of different sequence types. Oncotarget. 2016;7:82112-22.

21. Li Z, Tan C, Lin J, Feng Y. Diversified variants of the mcr-1-carrying plasmid reservoir in the swine lung microbiota. Sci China Life Sci. 2016;59:971-3.

22. Ye H, Li Y, Li Z, Gao R, Zhang H, et al. Diversified mcr-1-harbouring plasmid reservoirs confer resistance to colistin in human gut microbiota. MBio. 2016; 7:e00177. doi:10.1128/mBio.00177-16.

23. Doumith M, Godbole G, Ashton P, Larkin L, Dallman T, et al. Detection of the plasmid-mediated $\mathrm{mcr}-1$ gene conferring colistin resistance in human and food isolates of Salmonella enterica and Escherichia coli in England and Wales. J Antimicrob Chemother. 2016;71:2300-5.

24. Li XP, Fang LX, Song JQ, Xia J, Huo W, et al. Clonal spread of mcr-1 in PMQR-carrying ST34 Salmonella isolates from animals in China. Sci Rep. 2016;6:38511.

25. Stoesser N, Mathers AJ, Moore CE, Day NPJ, Crook DW. Colistin resistance

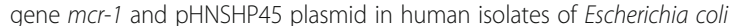
and Klebsiella pneumoniae. Lancet Infect Dis. 2016;16:285-6.

26. Zhao F, Feng Y, Lu X, McNally A, Zong Z. An IncP plasmid carrying the colistin resistance gene $m c r-1$ in Klebsiella pneumoniae from hospital sewage. Antimicrob Agents Chemother. 2016;61(2). doi: 10.1128/AAC. 02229-16.

27. Zeng KJ, Doi Y, Patil S, Huang X, Tian GB. Emergence of plasmid-mediated mcr-1 gene in colistin-resistant Enterobacter aerogenes and Enterobacter cloacae. Antimicrob Agents Chemother. 2016;60:3862-3.

28. Zhao F, Zong Z. Kluyvera ascorbata strain from hospital sewage carrying the mcr-1 colistin resistance gene. Antimicrob Agents Chemother. 2016;60: 7498-501.

29. Li XP, Fang LX, Jiang $P$, Pan D, Xia J, et al. Emergence of the colistin resistance gene $\mathrm{mcr}-1$ in Citrobacter freundii. Int J Antimicrob Agents. 2017; 49:786-7.

30. Sennati S, Di Pilato V, Riccobono E, Di Maggio T, Villagran AL, et al. Citrobacter braakii carrying plasmid-borne $\mathrm{mcr}$-1 colistin resistance gene from ready-to-eat food from a market in the Chaco region of Bolivia. J Antimicrob Chemother. 2017. doi:10.1093/jac/dkx078.
31. El Garch F, Sauget M, Hocquet D, LeChaudee D, Woehrle F, et al. mcr-1 is borne by highly diverse Escherichia coli isolates since 2004 in foodproducing animals in Europe. Clin Microbiol Infect. 2016;23(1):51.e1-4. doi:10.1016/j.cmi.2016.08.033. Epub 2016 Sep 8.

32. Delgado-Blas JF, Ovejero CM, Abadia Patino L, Gonzalez-Zorn B. Coexistence of $\mathrm{mcr}-1$ and blaNDM-1 in Escherichia coli from Venezuela. Antimicrob Agents Chemother. 2016;60:6356-8

33. Zheng B, Dong H, Xu H, Lv J, Zhang J, et al. Coexistence of MCR-1 and NDM-1 in clinical Escherichia coli isolates. Clin Infect Dis. 2016;63:1393-5.

34. Mediavilla JR, Patrawalla A, Chen L, Chavda KD, Mathema B, et al. Colistinand carbapenem-resistant Escherichia coli harboring mcr-1 and blaNDM-5, causing a complicated urinary tract infection in a patient from the United States. MBio. 2016;7(4). doi:10.1128/mBio.01191-16.

35. Yang RS, Feng Y, Lv XY, Duan JH, Chen J, et al. Emergence of NDM-5 and MCR-1-producing Escherichia coli clone ST648 and ST156 from a single Muscovy duck (Cairina moschata). Antimicrob Agents Chemother. 2016; 60(11):6899-902.

36. Yao X, Doi Y, Zeng L, Lv L, Liu JH. Carbapenem-resistant and colistinresistant Escherichia coli co-producing NDM-9 and MCR-1. Lancet Infect Dis. 2016;16:288-9.

37. Zhang $\mathrm{H}$, Seward $\mathrm{CH}$, Wu Z, Ye H, Feng Y. Genomic insights into the ESBL and MCR-1-producing ST648 Escherichia coli with multi-drug resistance. Sci Bull (Beijing). 2016;61:875-8.

38. Yang YQ, Zhang AY, Ma SZ, Kong LH, Li YX, et al. Co-occurrence of mcr-1 and ESBL on a single plasmid in Salmonella enterica. J Antimicrob Chemother. 2016;71:2336-8

39. Li A, Yang Y, Miao M, Chavda KD, Mediavilla JR, et al. Complete sequences of mcr-1-harboring plasmids from extended-spectrum-beta-lactamase- and carbapenemase-producing Enterobacteriaceae. Antimicrob Agents Chemother. 2016:60:4351-4.

40. Sun J, Li XP, Yang RS, Fang LX, Huo W, et al. Complete nucleotide sequence of an Incl2 plasmid coharboring blaCTX-M-55 and mcr-1. Antimicrob Agents Chemother. 2016;60:5014-7.

41. Gao R, Wang Q, Li P, Li Z, Feng Y. Genome sequence and characteristics of plasmid pWH12, a variant of the mcr-1-harbouring plasmid pHNSHP45, from the multi-drug resistant E. coli. Virulence. 2016:7:732-5.

42. Guo Q, Su J, McElheny CL, Stoesser N, Doi Y, et al. IncX2 and IncX1-X2 hybrid plasmids coexisting in FosA6-producing Escherichia coli. Antimicrob Agents Chemother. 2017. doi: 10.1128/AAC.00536-17. [Epub ahead of print].

43. Sun J, Yang RS, Zhang Q, Feng Y, Fang LX, et al. Co-transfer of bla $a_{N D M-5}$ and mcr-1 by an IncX3-X4 hybrid plasmid in Escherichia coli. Nat Microbiol. 2016; 1:16176.

44. Fernandes MR, McCulloch JA, Vianello MA, Moura Q, Perez-Chaparro PJ, et al. First report of the globally disseminated IncX4 plasmid carrying the mcr-1 gene in a colistin-resistant Escherichia coli sequence type 101 isolate from a human infection in Brazil. Antimicrob Agents Chemother. 2016;60: 6415-7.

45. Sun J, Fang LX, Wu Z, Deng $H$, Yang RS, et al. Genetic analysis of the Inc X4 plasmids: implications for a unique pattern in the mcr-1 acquisition. Sci Rep. $2017 ; 7: 424$

46. Zhi C, LV L, Yu LF, Doi Y, Liu JH. Dissemination of the $m c r-1$ colistin resistance gene. Lancet Infect Dis. 2016;16:292-3.

47. McGann P, Snesrud E, Maybank R, Corey B, Ong AC, et al. Escherichia coli harboring $\mathrm{mcr}-1$ and blaCTX-M on a novel IncF plasmid: first report of $\mathrm{mcr}-1$ in the United States. Antimicrob Agents Chemother. 2016;60:4420-1.

48. Xavier BB, Lammens C, Butaye P, Goossens H, Malhotra-Kumar S. Complete sequence of an IncFIl plasmid harbouring the colistin resistance gene $\mathrm{mcr}-1$ isolated from Belgian pig farms. J Antimicrob Chemother. 2016;71:2342-4

49. Zurfluh K, Klumpp J, Nuesch-Inderbinen M, Stephan R. Full-length nucleotide sequences of $\mathrm{mcr}$-1-harboring plasmids isolated from extendedspectrum-beta-lactamase-producing Escherichia coli isolates of different origins. Antimicrob Agents Chemother. 2016;60:5589-91.

50. Luo H, Zhang CT, Gao F. Ori-Finder 2, an integrated tool to predict replication origins in the archaeal genomes. Front Microbiol. 2014;5:482.

51. Laslett D, Canback B. ARAGORN, a program to detect tRNA genes and tmRNA genes in nucleotide sequences. Nucleic Acids Res. 2004; 32:11-6.

52. Lagesen $\mathrm{K}$, Hallin $\mathrm{P}$, Rodland EA, Staerfeldt HH, Rognes T, et al. RNAmmer: consistent and rapid annotation of ribosomal RNA genes. Nucleic Acids Res. 2007;35:3100-8 
53. Ou HY, He X, Harrison EM, Kulasekara BR, Thani AB, et al. MobilomeFINDER: web-based tools for in silico and experimental discovery of bacterial genomic islands. Nucleic Acids Res. 2007;35:W97-104.

54. Carattoli A, Zankari E, Garcia-Fernandez A, Voldby Larsen M, Lund O, et al. In silico detection and typing of plasmids using PlasmidFinder and plasmid multilocus sequence typing. Antimicrob Agents Chemother. 2014;58:3895-903.

55. Varani AM, Siguier P, Gourbeyre E, Charneau V, Chandler M. ISsaga is an ensemble of web-based methods for high throughput identification and semi-automatic annotation of insertion sequences in prokaryotic genomes. Genome Biol. 2011;12:R30.

56. Siguier P, Perochon J, Lestrade L, Mahillon J, Chandler M. ISfinder: the reference centre for bacterial insertion sequences. Nucleic Acids Res. 2006; 34:D32-6.

57. Arndt D, Grant JR, Marcu A, Sajed T, Pon A, et al. PHASTER: a better, faster version of the PHAST phage search tool. Nucleic Acids Res. 2016;44:W16-21.

58. Fouts DE. Phage_Finder: automated identification and classification of prophage regions in complete bacterial genome sequences. Nucleic Acids Res. 2006;34:5839-51.

59. Liu B, Pop M. ARDB — antibiotic resistance genes database. Nucleic Acids Res. 2009;37:D443-7.

60. McArthur AG, Waglechner N, Nizam F, Yan A, Azad MA, et al. The comprehensive antibiotic resistance database. Antimicrob Agents Chemother. 2013:57:3348-57.

61. Kleinheinz KA, Joensen KG, Larsen MV. Applying the ResFinder and VirulenceFinder web-services for easy identification of acquired antibiotic resistance and $\mathrm{E}$. coli virulence genes in bacteriophage and prophage nucleotide sequences. Bacteriophage. 2014;4:e27943.

62. Sekizuka T, Kawanishi M, Ohnishi M, Shima A, Kato K, et al. Elucidation of quantitative structural diversity of remarkable rearrangement regions, shufflons, in Incl2 plasmids. Sci Rep. 2017;7:928.

63. Wang Q, Sun J, Ding Y, Li XP, Liu YH, et al. Genomic insights into mcr-1positive plasmids carried by the colistin-resistant Escherichia coli from the inpatients. Antimicrob Agents Chemother. 2017;61(7). doi:10.1128/AAC. 00361-17. [Epub ahead of print].

\section{Submit your next manuscript to BioMed Central and we will help you at every step:}

- We accept pre-submission inquiries

- Our selector tool helps you to find the most relevant journal

- We provide round the clock customer support

- Convenient online submission

- Thorough peer review

- Inclusion in PubMed and all major indexing services

- Maximum visibility for your research

Submit your manuscript at www.biomedcentral.com/submit 